\title{
In vivo functional differences in microvascular response of 4T1 and Caki-1 tumors after treatment with OXi4503
}

\author{
MAMTA WANKHEDE ${ }^{1}$, CASEY DEDEUGD ${ }^{1}$, DIETMAR W. SIEMANN ${ }^{2}$ and BRIAN S. SORG ${ }^{1}$ \\ ${ }^{1}$ J. Crayton Pruitt Family Department of Biomedical Engineering and ${ }^{2}$ Department of \\ Radiation Oncology, University of Florida, Gainesville, FL 32611, USA
}

Received August 14, 2009; Accepted October 8, 2009

DOI: 10.3892/or_00000685

\begin{abstract}
T1 mouse mammary adenocarcinomas and Caki-1 human renal cell carcinomas grown in mouse dorsal window chambers were serially treated with the vascular disrupting agent (VDA) OXi4503 and their responses compared. The real-time in vivo response was assessed using spectral imaging of microvascular hemoglobin saturation. To our knowledge this is the first use of spectral imaging technology for investigation of vascular disrupting agents. Previous research showing tumor size dependence in the treatment response to VDAs suggested that for the size of tumors used in this study only a moderate response would be observed; however, the tumors unexpectedly had very different responses to treatment. Caki-1 tumors showed little permanent vessel damage and experienced transient vessel collapse with time-dependent oxygenation changes followed by recovery starting at $6 \mathrm{~h}$ after treatment. Caki-1 tumors did not manifest necrotic avascular regions even after repeated treatments. These results are consistent with those obtained using other imaging modalities and histology. In contrast, similarly sized 4T1 tumors showed extensive vessel disintegration, minor vascular collapse, and a drop in tumor oxygenation up to $6 \mathrm{~h}$ post-treatment, after which reperfusion of collapsed vessels and extensive vascular remodeling and neovascularization of the tumor rim occurred from 8-48 h. The completely disintegrated vessels did not recover and left behind avascular and apparently necrotic regions in the tumor core. Spectral imaging appears to be a useful technique for in vivo investigation of vascular disrupting agents. The differential responses of these two tumor-types suggest that further investigation of the mechanisms of action of VDAs and individual characterization of tumor VDAresponses may be needed for optimal clinical use of these agents.
\end{abstract}

Correspondence to: Dr Brian S. Sorg, J. Crayton Pruitt Family Department of Biomedical Engineering, 130 BME Building, PO Box 116131, University of Florida, Gainesville, FL 32611, USA E-mail: brian.sorg@bme.ufl.edu

Key words: angiogenesis, oxygen, spectral imaging, vascular targeting, window chamber

\section{Introduction}

The significance of tumor microvasculature for tumor growth, proliferation and metastasis has been recognized and numerous methods have been developed to impair tumor vessels with the intent of cutting off the tumor blood supply and access to oxygen and nutrients. An array of specific tumor vessel targeting strategies emerged after Denekamp et al distinguished tumor endothelial cells from normal tissue endothelial cells based on their significantly higher proliferation rate (1-3). Currently, a number of vascular targeting approaches are in development for selective and specific targeting of the tumor vessels while sparing the normal tissue vasculature (4-9).

Vascular disrupting agents (VDAs) damage existing tumor blood vessels while antiangiogenic therapies such as bevacizumab inhibit new blood vessel formation (10). Combretastatins, initially derived from the South African tree Combretum caffrum, represent a class of potent tubulin binding agents with significant vascular disrupting activity $(5,8)$. Lead agents Combretastatin A4-P (CA4-P) and OXi4503 (CA1-P) have been shown to result in time-dependent decreases in tumor perfusion, increased tumor vascular permeability, and significant tumor vascular damage in a wide variety of preclinical tumor models $(8,11-13)$. In the clinic, these agents have now entered Phase II/III evaluation $(14,15)$.

Despite extensive tumor destruction following VDA therapy, a hallmark of VDA treatment is the survival of neoplastic cells at the tumor periphery (14). This surviving 'viable rim' can lead to subsequent tumor reperfusion and regrowth following drug treatment $(11,12)$. In preclinical models and some clinical studies, functional and morphological parameters such as tumor perfusion, blood flow, vascular structure, permeability and vascular damage are used to evaluate the effect of OXi4503 and CA4-P on tumor and normal tissue $(11,16-19)$. Some of the methods used cannot furnish continuous real-time in vivo information as they require animal euthanasia at the respective time-points and do not permit serial follow-up of the same tumor after VDA treatment. Hence, several different techniques are used for the in vivo assessment of VDA-induced functional and morphological changes in murine and human tumor vasculature. For example, Sheng et al used microsphere fluorescence to assess in vivo tumor blood flow in murine flank tumors after OXi4503 treatment (13). Other techniques such as magnetic resonance imaging and positron emission tomo- 
graphy can provide functional information regarding blood flow, tumor perfusion, and vascular permeability $(12,20)$, however, with poor microvascular resolution. In vivo microscopy techniques such as multi-photon fluorescence microscopy in a window chamber model have been employed by Tozer et al which provides vascular function data with improved microvessel resolution (21), and can be used to simultaneously observe serial morphological changes after VDA treatment.

Spectral imaging is an effective tool for measurement of microvessel vessel oxygenation in tumor microvasculature (22-24). The oxygenation of a vessel depends on several factors such as hemoglobin saturation (HbSat), perfusion and red cell flux $(22,25)$. In the present study, spectral imaging is used with mouse dorsal skin-fold window chambers to measure microvascular functional changes in terms of HbSat in two different tumors, a mouse mammary carcinoma (4T1) and human renal cell carcinoma (Caki-1), during serial treatments with the VDA OXi4503.

\section{Materials and methods}

Tumor cells. 4T1 mouse mammary adenocarcinoma cells (a gift from Mark W. Dewhirst, Duke University Medical Center, Durham, NC) were cultured in DMEM (Cellgro, Inc., $1 \mathrm{X}, 4.5 \mathrm{~g} / \mathrm{l}$ glucose, L-glutamine and sodium pyruvate) containing $10 \%$ fetal bovine serum (Biowhittaker, Inc.), $1 \%$ L-glutamine (Clonogen, Inc.) and $1 \%$ penicillin streptomycin (Clonogen, Inc.). A single cell suspension of approximately $5 \times 10^{3}$ to $10 \times 10^{3}$ cells prepared in serum-free DMEM was used to initiate tumors in window chambers of the experimental mice. Caki-1 tumor xenografts (18) were initiated in a single hind limb of donor mice by inoculating with $2 \times 10^{5}$ to $5 \times 10^{5}$ tumor cells. Tumors were excised when they reached about $200 \mathrm{~mm}^{3}$ in size, and a single cell suspension was prepared in DMEM. About $1 \times 10^{6}$ cells were used to initiate a window chamber tumor in the experimental mice.

Animal model. All in vivo procedures were conducted in accordance with a protocol approved by University of Florida Institutional Animal Care and Use Committee. Athymic (nu/nu) female mice weighing at least $21 \mathrm{~g}$ (Charles River Laboratories, Raleigh, NC) were surgically implanted with a titanium window chamber on the dorsal skin flap. During surgery mice were anesthetized by an IP injection of ketamine $(100 \mathrm{mg} / \mathrm{kg})$ and xylazine $(10 \mathrm{mg} / \mathrm{kg})$. A tumor was initiated in the window chamber during surgery by injecting the tumor cells subcutaneously in the dorsal skin flap prior to placing a 12-mm diameter no. 2 round glass coverslip (Erie Scientific, Portsmouth, NH) over the exposed skin. After surgery animals were housed in an environmental chamber maintained at $33^{\circ} \mathrm{C}$ and $50 \%$ humidity with free access to food and water and standard 12-h light/dark cycles.

Drug preparation and experimental design. OXi4503, a gift from OXiGENE, Inc. (Waltham, MA) was administered at a dose of $10 \mathrm{mg} / \mathrm{kg}$ IP ( $0.01 \mathrm{ml} / \mathrm{g}$ of mouse body weight). The light-sensitive drug was protected from light during handling. OXi4503 was suspended in a solution of sterile saline and $50 \mu \mathrm{l}$ per $\mathrm{ml}$ of $5 \%$ sodium carbonate solution before injection.
The drug was prepared fresh daily. Control groups received an equivalent volume of sterile saline and $5 \%$ sodium carbonate solution. The tumors were allowed to grow for 78 days and the treatment was started when tumors reached a size of about 3-4 mm. Spectral imaging was performed immediately prior to and post-treatment, and also at 2, 4, 6, 8,24 and $48 \mathrm{~h}$ post-treatment. After the 48 -h imaging timepoint the mouse was re-treated and the same imaging schedule was maintained. The treatment was repeated 3-5 times on each of the experimental mice. In all, 5 mice bearing 4T1 tumors were treated with $\mathrm{OXi} 4503$ and 3 mice were carrieronly controls. For Caki-1 tumors the OXi4503 treatment was performed on 5 mice, while 2 mice received carrier only minus drug.

Imaging system, image acquisition and image processing. The spectral imaging system, image acquisition, and image processing methods for hemoglobin saturation measurements were discussed in detail previously (24). Briefly, a Zeiss AxioImager microscope (Carl Zeiss, Inc., Thornwood, NY) was used as a basic platform for the imaging system. For transillumination images, a 100-W tungsten halogen lamp was used. The long working distance objectives used were $2.5 \mathrm{X}$ and 5X Fluars, 10X EC Plan-NeoFluar and 20X LD-PlanNeoFluar (Carl Zeiss, Inc.). For spectral information, brightfield transmitted light images were obtained using a CCD camera thermoelectrically cooled to $-20^{\circ} \mathrm{C}$ (DVC Co., Austin, TX; Model no.1412AM-T2-FW). Band-limited optical filtering was accomplished using a C-mounted liquid crystal tunable filter (CRI, Inc., Woburn, MA) with a 400- to 720-nm transmission range and a $10-\mathrm{nm}$ nominal bandwidth, placed in front of the CCD camera. LabVIEW8 (National Instruments Corp., Austin, TX) was used to automatically control the tuning of the LCTF and acquire images with the CCD camera. Microvessel hemoglobin saturation measurements and images were created from the spectral image data $(24,26)$.

\section{Results}

During each treatment the tumor vasculature experienced two distinct phases: a destruction phase closely followed by a recovery phase. The treatment resulted in a combination of three major effects on the tumor vasculature: i) transient vessel collapse, ii) permanent vessel disintegration or iii) no structural damage with only temporary functional loss. Transient vessel collapse refers to the temporary disappearance of the tumor core vessels during the destruction phase of each treatment. These original tumor vessels re-emerged during the recovery phase and regained their functionality. Tumor core vasculature that underwent vessel disintegration never reappeared and in some cases there was evidence of hemorrhaging. Some tumor vessels as well as the normal vasculature did not suffer from structural damage but still manifested temporary oxygenation changes.

Treatment of 4T1 mouse mammary carcinomas. Each 4T1 tumor-bearing mouse $(n=5)$ received 3 serial treatments of OXi4503 administered every 48 h. Fig. 1 shows a typical 4T1 tumor treatment progression. Prior to OXi4503 administration, the tumor vessels as well as the normal vasculature 


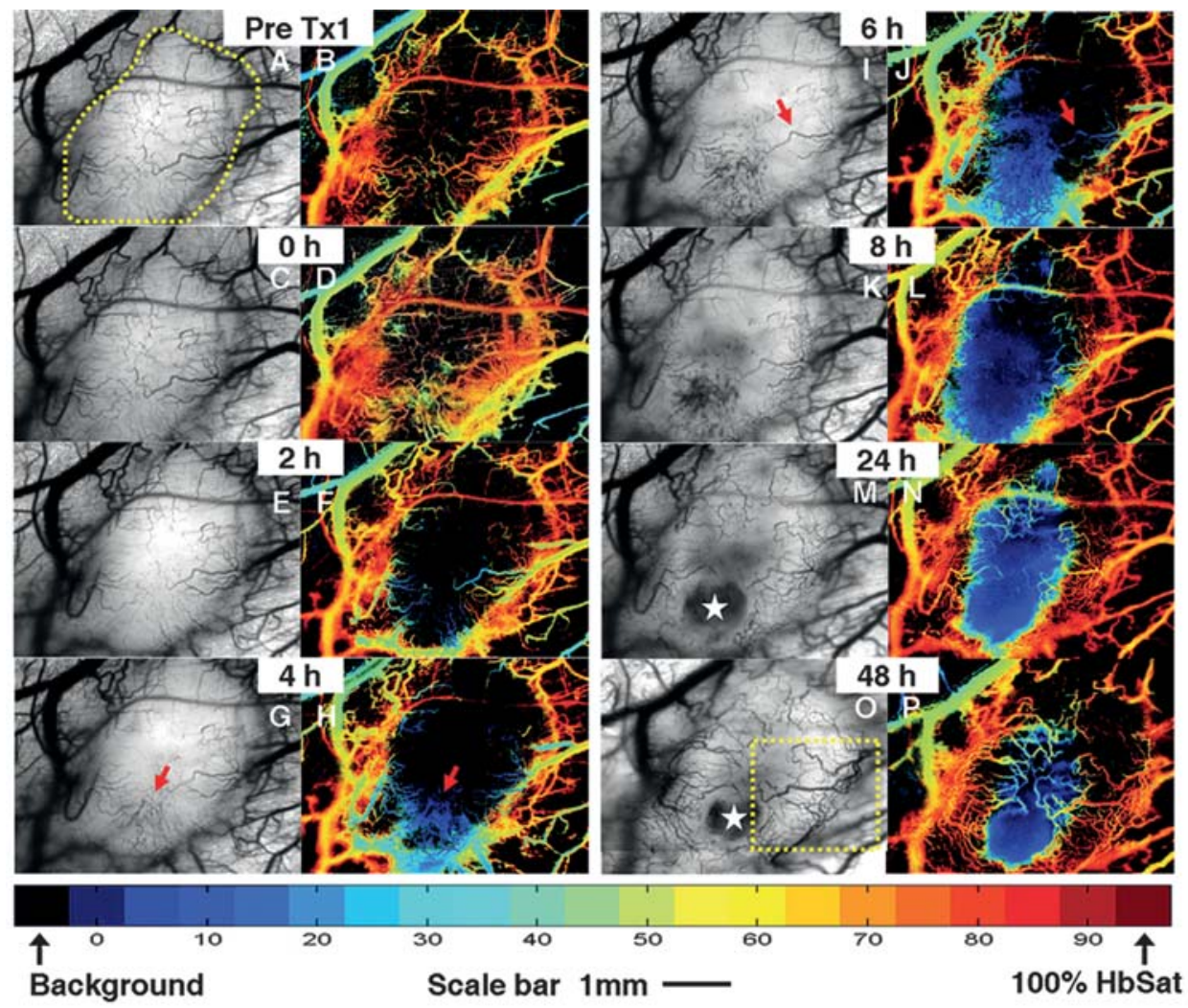

Figure 1. Typical OXi4503 treatment progression in a 4T1 tumor-bearing mouse. The brightfield images (left hand figures in each panel) show structural alterations in the vasculature through the course of the single treatment. The approximate tumor border is indicated by the dotted line in (A). Vessels outside the dotted line are considered normal tissue vasculature. The tumor periphery consisted of a $250-$ to $500-\mu \mathrm{m}$ thin border on the circumference of the tumor. Remaining tumor vasculature except for the periphery constituted the tumor core. Images were obtained at x 2.5 magnification with image dimensions of $4.15 \times 3.125 \mathrm{~mm}$. The HbSat maps are shown in the right hand figures in each panel. The oxygenation levels in the HbSat maps are color coded as indicated by the colorbar. Arrows in frames $(\mathrm{G})$ and $(\mathrm{H})$ highlight the disintegrating vasculature, while arrows in (I) and (J) indicate an example vessel that persists through all time-points with only oxygenation changes. The star in frames $(\mathrm{M})$ and $(\mathrm{O})$ indicate the avascular regions created by the OXi4503 treatment.

were intact and functional with ample oxygenated blood supply (Fig. 1A, B). At 2-h post OXi4503 treatment, structural collapse of the tumor vasculature was observed (Fig. 1E) accompanied with significant reduction in oxygenation (Fig. 1F) with initiation of minor vessel breakdown in some areas of the tumor. Progressive vessel disintegration advanced from the tumor core towards the periphery between 2-8 $\mathrm{h}$ post-treatment resulting in functional loss in the tumor core (disintegrated vascular network highlighted in Fig. 1G, $\mathrm{H})$. Some tumor vessels did not exhibit permanent structural alterations and instead temporarily lost functionality (example vessel highlighted in Fig. 1I, J). As a result of the structural breakdown, the oxygenation in the tumor vasculature plummeted rapidly up to $6 \mathrm{~h}$ after treatment.

From 8-48 h post-treatment, a rapid structural and functional recovery ensued, progressing from the tumor periphery towards the tumor core. The vessels which had temporarily lost functionality without losing their structure were gradually reoxygenated (Fig. 1I, J-O, P). The collapsed tumor vessels re-emerged and reperfused (Fig. 1M, N-O, P) whereas the vessels in which the structure was completely disintegrated did not recover (Fig. 1M, O). Rapid neovascularization and vascular remodeling was observed between $24-48 \mathrm{~h}$ after OXi4503 administration in the form of new vessel sprouts and new network interconnections. Fig. 2 gives a magnified view of the vessel remodeling and neovascularization in the tumor periphery during the recovery phase from $8-48 \mathrm{~h}$ after a treatment.

Regional quantification of the vessel hemoglobin saturation was performed by dividing the 2-D image vasculature into tumor core, tumor periphery, or normal tissue regions. For example, the tumor nodule is indicated enclosed by the dotted line in Fig. 1A. Vessels outside the dotted line were considered as normal tissue vasculature. The tumor periphery vessels consisted of the vessels in the $250-500 \mu \mathrm{m}$ thin border on the circumference of the tumor nodule. The remaining tumor vessels constituted the tumor core vasculature.

Fig. 3A shows the tumor before and after each of the serial treatments. An increasing fraction of vasculaturedeprived apparently necrotic tumor area was observed with every serial treatment. In Fig. 3B is a cumulative plot for three serial treatments in five mice showing the HbSat changes over time. Two individual treatments from two different mice have been omitted from the data in Fig. 3B owing to lack of considerable oxygenation changes. As seen in Fig. 3B, the core vessels experienced the greatest impact of the treatment followed by the periphery and the normal vessels. Interestingly, the adjacent normal vasculature also experienced oxygenation changes even though it remained structurally intact throughout the treatment. From 2-6 h there was a steep decrease in all three region vessels with an onset of steady recovery from $8 \mathrm{~h}$ onward. The normal and tumor periphery 


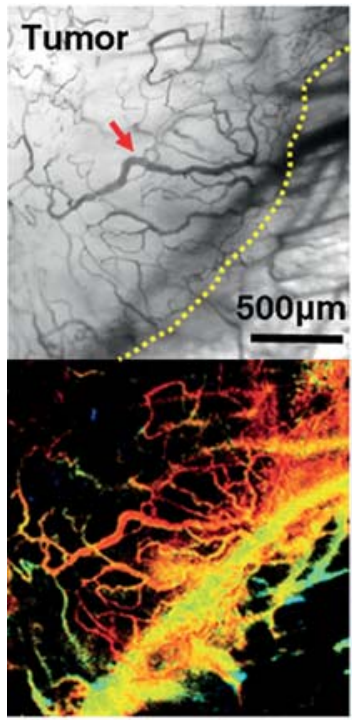

Pre Tx1

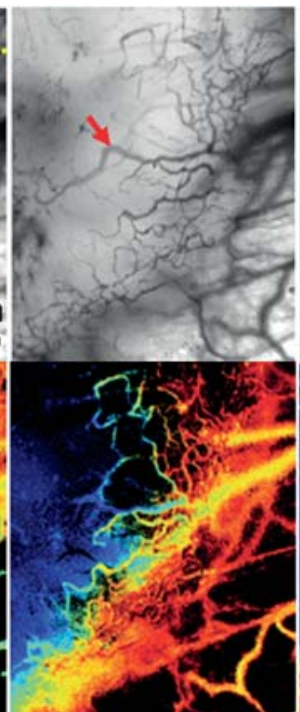

$8 \mathrm{~h}$

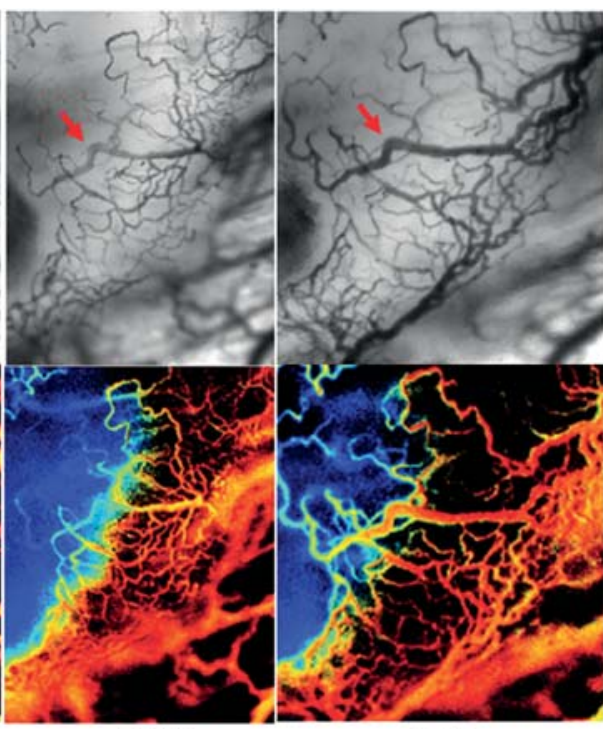

$48 \mathrm{~h}$

Figure 2. Recovery, reoxygenation, vascular remodeling, and neovascularization in the 4T1 tumor during the recovery phase after OXi4503 treatment for the area indicated in Fig. 10 by the dotted rectangle. The approximate tumor border is demarcated by the dotted line in the first bright field image. The arrows highlight an example vessel that maintains its structure throughout the treatment but undergoes dramatic oxygenation changes. The colorscale for hemoglobin saturation is the same as in Fig. 1.

A
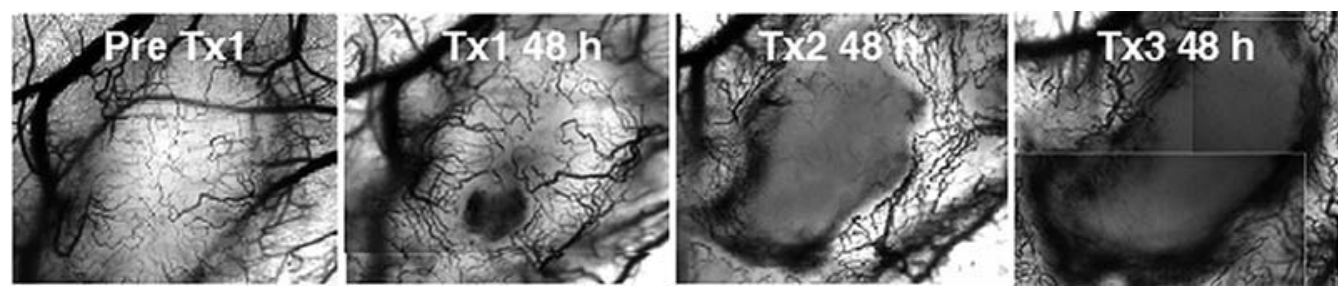

B

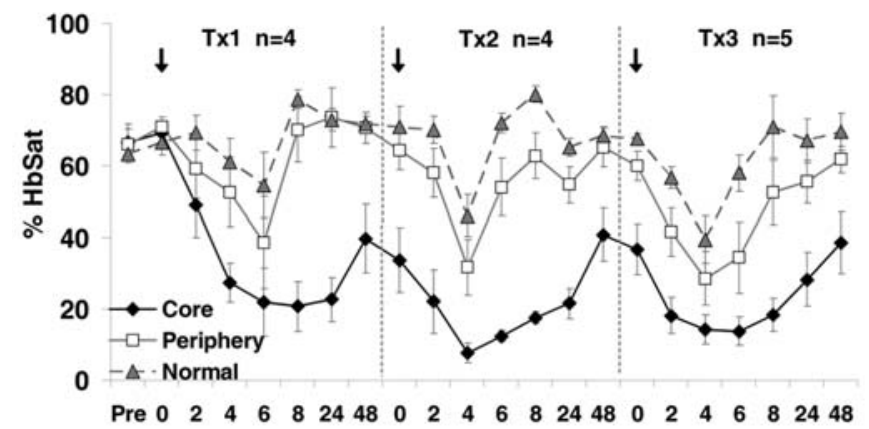

C

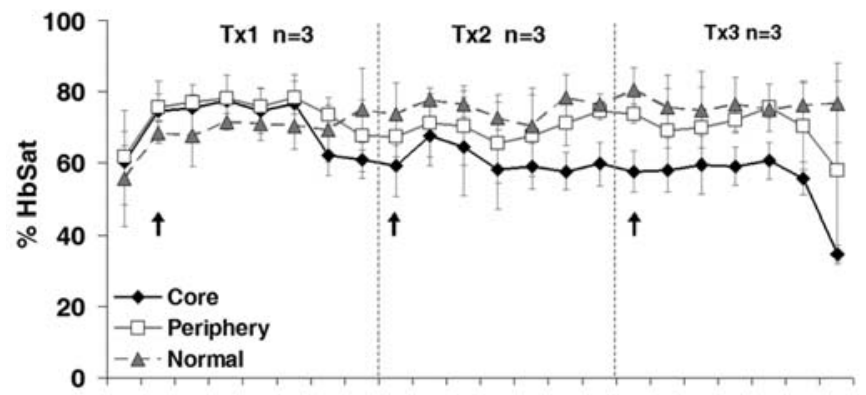

Pre $\begin{array}{lllllllllllllllllllll}0 & 2 & 4 & 6 & 8 & 24 & 48 & 0 & 2 & 4 & 6 & 8 & 24 & 48 & 0 & 2 & 4 & 6 & 8 & 24 & 48\end{array}$

Figure 3. Quantitative tumor regional analysis for 4T1 tumor treatment. The tumor was divided into core, periphery, and normal tissue for purposes of analysis. (A) Brightfield images before and after every successive treatment of the tumor in Fig. 1. (B) Cumulative HbSat values (mean \pm standard error) for 3 serial treatments in all $54 \mathrm{~T} 1$ tumor mice. Arrows indicate drug administration. (C) Cumulative HbSat values (mean \pm standard error) for 3 serial sham treatments (saline) in all 3 control mice.

vessels seemed to have an earlier and almost complete recovery back to the original oxygenation values, as opposed to the tumor core vessel oxygenation. At 24 and $48 \mathrm{~h}$ posttreatment normal and tumor periphery regions were highly oxygenated but the tumor core did not recover as much and remained relatively less oxygenated. Interestingly, in one mouse the first of the three serial treatments was unable to elicit noticeable structural alterations with only subtle oxygenation changes, while in another mouse the second of the three serial treatments failed to produce any observable morphological or functional changes. Moreover, the later treatments in both these mice were able to produce profuse vascular damage with vivid changes in oxygenation.

No microvessel structural or functional changes were observed in the control mice administered carrier-only treatment. In controls the tumor mass and vasculature continued to develop unrestrained. Over the entire experimental period, the tumor core region gradually becomes less oxygenated 


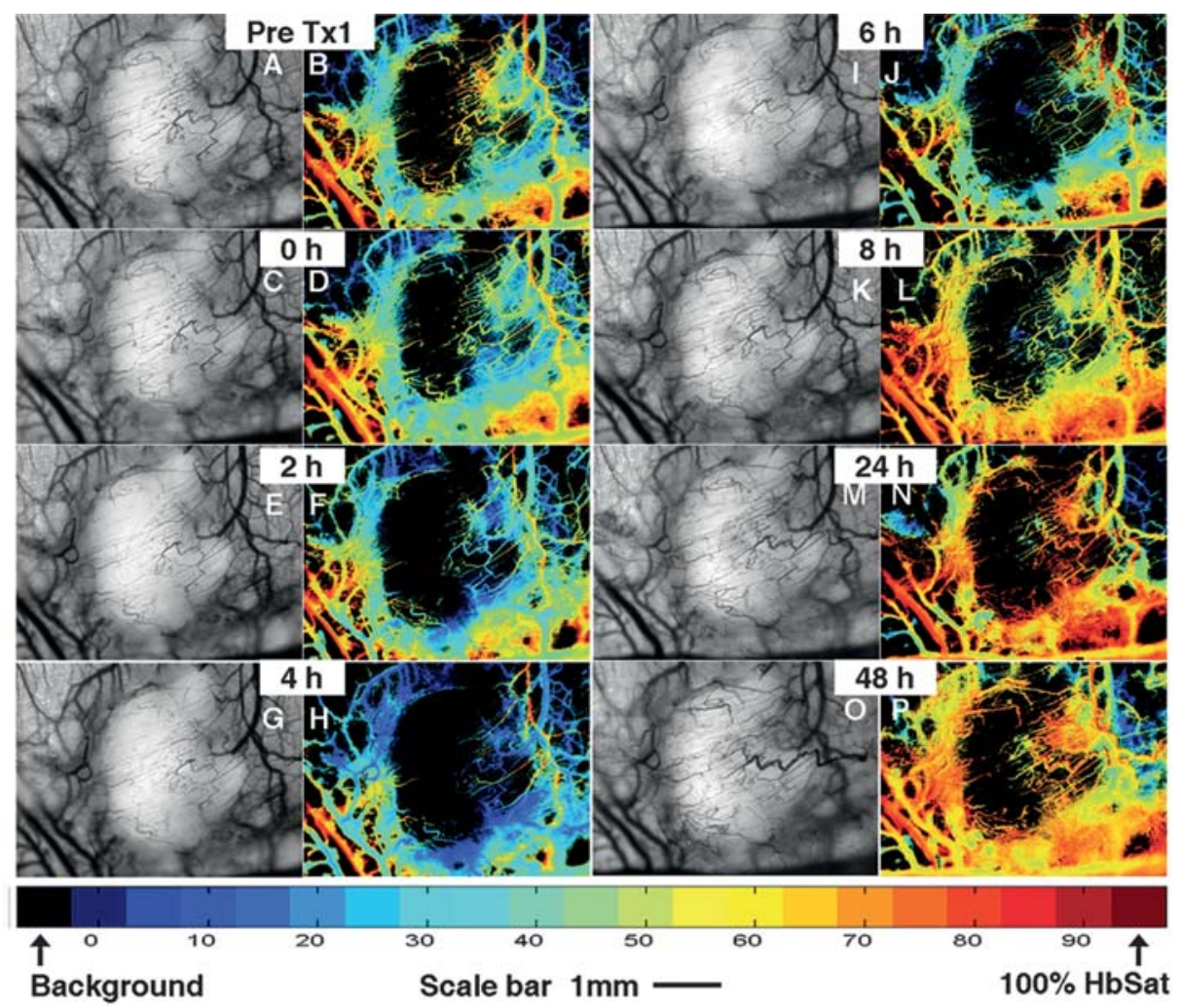

Figure 4. OXi4503 treatment 2 of a Caki-1 tumor bearing mouse. Images were obtained at x 2.5 magnification with image dimensions of $4.15 \times 3.125$ mm. The HbSat maps are shown in the right hand figures in each panel. The oxygenation levels in the HbSat maps are color coded as indicated by the colorbar.

A

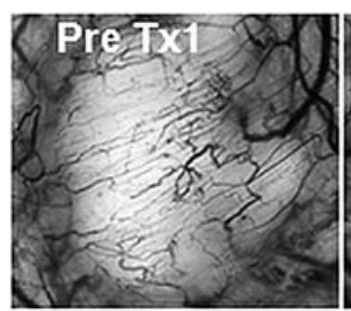

B

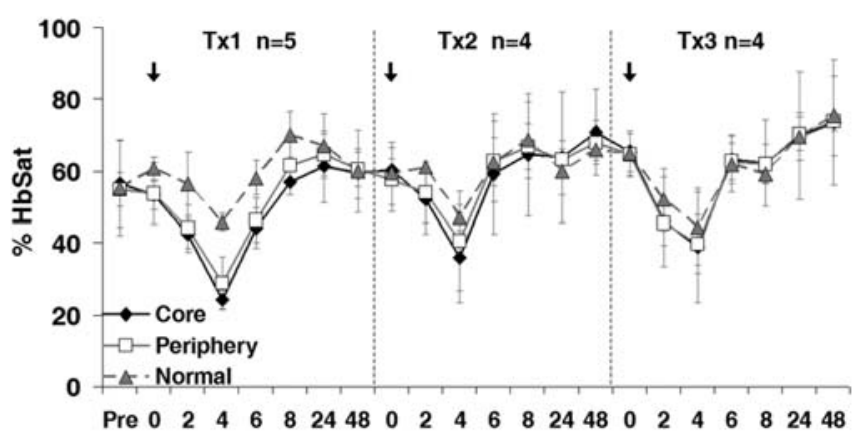

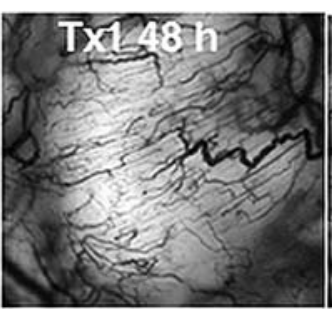
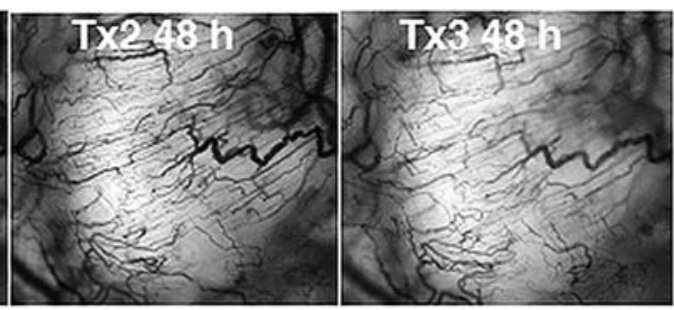

C

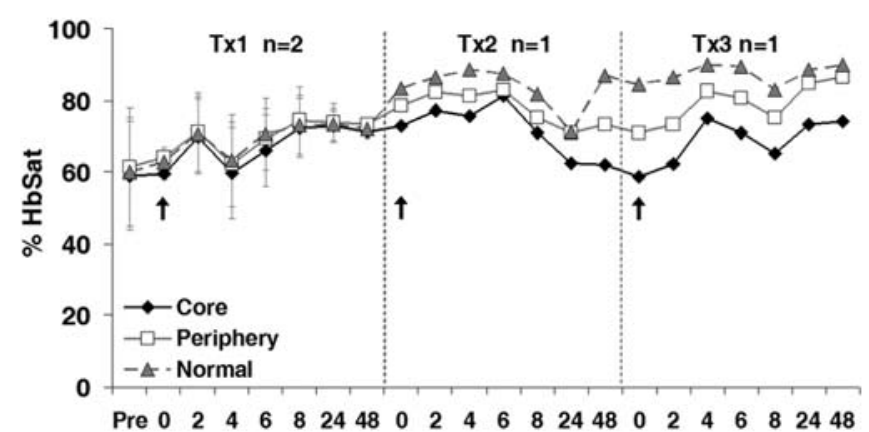

Figure 5. Quantitative tumor regional analysis for Caki-1 tumor treatment. The tumor was divided into core, periphery, and normal tissue for purposes of analysis. (A) Brightfield images before and after every successive treatment of the tumor in Fig. 4. (B) Cumulative HbSat values (mean \pm standard error) for 3 serial treatments in all $54 \mathrm{~T} 1$ tumor mice. Arrows indicate drug administration. (C) Cumulative HbSat values (mean \pm standard error) for 3 serial sham treatments (saline) in a control mouse.

compared to the tumor periphery and normal tissue regions as the tumor expands. Fig. 3C shows the hemoglobin saturation quantification results for carrier-only treatments in 3 mice.

Treatment of Caki-1 human renal carcinomas. An example OXi4503-treated Caki-1 tumor is shown in Fig. 4. A pattern of structural and functional changes including vascular collapse, vessel disintegration, and oxygenation changes after OXi4503 treatment were observed. Notably, compared to 4T1 tumors the Caki-1 tumors manifested more vessel collapse and lesser vascular disintegration. Among the total number of treatments $(n=18)$, only one single treatment in one mouse 


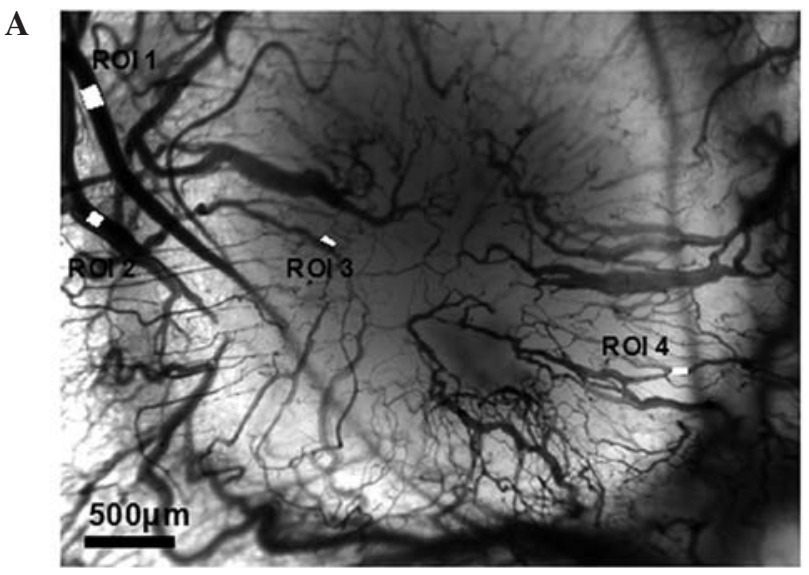

B

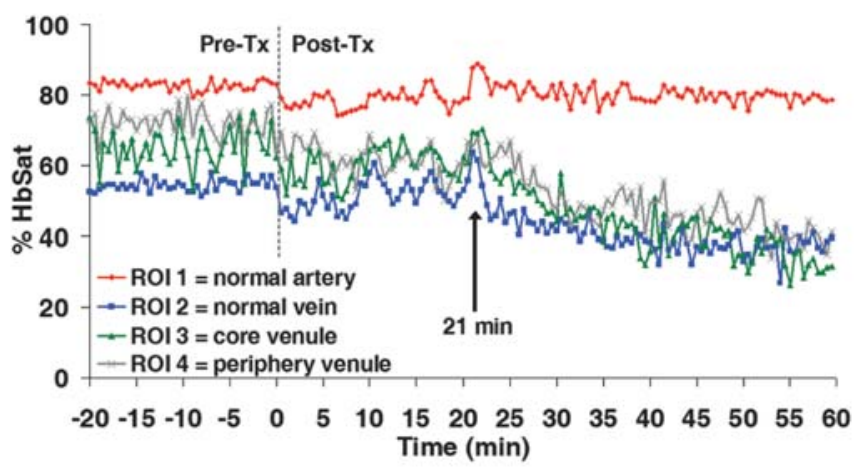

Figure 6. Rapid oxygenation changes in 4T1 tumor microvasculature after treatment with VDA OXi4503. The mouse was imaged for $20 \mathrm{~min}$ before treatment and for $60 \mathrm{~min}$ after treatment. Spectral images were obtained every $30 \mathrm{sec}$. (A) Brightfield image of the tumor microvasculature. (B) Hemoglobin saturation before and after OXi4503 treatment.

showed a comparable amount of tumor vessel disintegration to $4 \mathrm{~T} 1$ tumors. For the rest of the treatments, as shown in Fig. 4E, F-I, J, the tumor vasculature underwent a temporary vessel collapse from $2-4 \mathrm{~h}$ with minimal structural breakdown. The Caki-1 tumor vasculature recovered earlier with the tumor periphery gradually rejuvenated by $6 \mathrm{~h}$ posttreatment (Fig. 4I, J). During the recovery period from 6-48 h post-treatment, the collapsed vessels reappeared. Similar to 4T1 tumors, some Caki-1 tumor vessels remained intact but experienced dramatic oxygenation changes. Even though there was minimal permanent vessel damage in Caki-1 tumors as compared to 4T1 tumors, there were explicit changes in vessel hemoglobin saturations in tumor and normal vasculature as seen in Figs. 4 and 5. The brightfield images before and after each of the serial treatments is shown in Fig. 5A and the cumulative quantified vessel oxygenation during each of the serial treatments in five mice is shown in Fig. 5B. From Fig. 5B it can be seen that the tumor core in this tumor type did not have a decrease in microvessel oxygenation during the disruption phase as large in magnitude compared to $4 \mathrm{~T} 1$ tumors. The core vessel oxygenation was tightly coordinated with the tumor periphery and normal vessel oxygenation, and recovered as much and as fast as the latter two. From the brightfield images in Fig. 5A it can be seen that compared to 4T1 tumors (Fig. 3A) there was minimal vessel disintegration and no obvious avascular regions. The control mice which received the carrier-only treatment did not show any dramatic structural and functional changes as shown in Fig. 5C. With the increasing tumor mass, there was a gradient in the tumor vessel oxygenation from normal surroundings towards tumor core, with core vessels being the least oxygenated.

Even though there was no apparent necrosis evident in Caki-1 tumors, the tumor size seemed to be controlled by OXi4503 treatment. Tumor size in terms of apparent tumor area was measured from the 2-D brightfield images using ImageJ software (ImageJ 1.37a, Wayne Rasband, NIH, USA) and statistical size comparison between control and OXi4503treated tumors was performed. On average the untreated tumors were significantly larger at $48 \mathrm{~h}\left(4.91 \mathrm{~mm}^{2}, \mathrm{p}<0.03\right.$, two-tailed t-test: paired two sample for means) than the starting tumor size for the first sham treatment $\left(3.44 \mathrm{~mm}^{2}\right)$, and twice the starting size after a fourth sham treatment $\left(7.23 \mathrm{~mm}^{2}\right)$. In contrast, the OXi4503 treated tumors did not show any statistically significant increase from their original size at the start of treatment $(\mathrm{p}>0.36$, two-tailed t-test: paired two sample for means).

Rapid oxygenation changes after OXi4503 treatment. A mouse with a 4T1 tumor was imaged at 30 -sec intervals for 20 min prior to drug administration and 60 min after drug administration for real-time recording of the immediate microvascular effects of the drug. In this particular example (Fig. 6) significant effects began at about 21 min after administration. There was a clear reduction in oxygenation of the tumor periphery, core and normal draining venule. Only the feeding artery oxygenation remained relatively unchanged.

\section{Discussion}

OXi4503, a Combretastatin family member, is a tubulin binding drug $(27,28)$ and currently considered among the most potent VDAs $(13,29)$. In this study, we employed spectral imaging of window chamber tumors to investigate the realtime VDA-induced modulation in microvessel structure and function of two different tumor types during multiple serial treatments with OXi4503. Many of the observations with our imaging system were consistent with those reported by others using different techniques. For example, both 4T1 and Caki-1 tumors experienced a rapid drop in vascular oxygenation followed by recovery over $48 \mathrm{~h}$ post-treatment. The timings of our observations for vascular disruption and recovery are similar to those reported by Salmon and Siemann using Hoechst-33342 vessel staining for studying vascular shutdown and dynamic contrast-enhanced magnetic resonance imaging for quantifying tumor perfusion inhibition (12). Both these methods suggested $80-90 \%$ tumor perfusion reduction at $4 \mathrm{~h}$, with onset of recovery from $24-48 \mathrm{~h}(\sim 63 \%$ recovery at $48 \mathrm{~h}$ ). In our study the Caki-1 tumor recovery started earlier than 4T1 tumor recovery beginning at about $6 \mathrm{~h}$ posttreatment while $4 \mathrm{~T} 1$ recovery started around $8 \mathrm{~h}$ posttreatment. In previous studies using tumor histology to assess VDA effects, there were indirect indications of vessel hemorrhaging due to the presence of red blood cells in the tissue (30). With our system, direct real-time visualization of vessel hemorrhaging was possible and confirmed by hemoglobin 
saturation measurements that showed the presence of deoxygenated extravasated red blood cells. We observed rapid onset of microvessel functional changes in the form of decreasing hemoglobin saturation occurring mostly in tumor capillaries and draining venules starting about $21 \mathrm{~min}$ after administration of OXi4503, consistent with reported times for other VDAs (31).

Real-time functional imaging of serial OXi4503 treatments highlighted unexpected differences in the responses of the $4 \mathrm{~T} 1$ and Caki-1 tumors. The Caki-1 tumors were less responsive to vascular damage induced by OXi4503 than 4T1 tumors. The major Caki-1 tumor response was temporary vessel collapse with minimal vascular disruption followed by reperfusion and reoxygenation at later time-points during recovery. Only a small fraction of the tumor core vessels did not recover. This response was very reproducible with multiple serial treatments. In 4T1 tumors there was extensive permanent microvessel damage and more vessel damage occurred with each subsequent treatment. Consistent with the greater vascular breakdown in 4T1 tumors, there was greater deoxygenation in $4 \mathrm{~T} 1$ core vessels than in Caki-1 tumors. Despite the fact that in the Caki-1 tumors there was more temporary vessel collapse than permanent vessel disruption, treatment with OXi4503 seemed to control tumor growth similar to 4T1 tumors. It should be noted that while there was little permanent microvessel disruption and a lack of apparent necrosis in the Caki-1 tumors after OXi4503 administration, the treatments were highly dynamic and perturbative in terms of the acute effects on microvessel oxygenation.

Another difference in the tumor treatment response was the lack of apparent necrotic areas in the Caki-1 tumors even after repeat doses, while similarly sized 4T1 tumors developed what appeared to be extensive necrotic areas in the tumor core. The observed induction of necrosis in the 4T1 tumors was unexpected given previous research that showed size dependence in tumor response to VDA treatment. Siemann and Rojiani, using several different rodent and human tumor xenografts including Caki-1 tumors in mice, showed that larger tumors $(>1 \mathrm{~g})$ had significantly larger areas of necrosis on the order of $90 \%$ after treatment with ZD6126 than smaller tumors $(<0.3 \mathrm{~g}, \sim 25 \%$ necrosis $)$ treated with the same dose (32). The window chamber tumors fall into the smaller tumor size category, so the lack of apparent necrotic areas in the Caki-1 tumors in this study is consistent with the previous results with ZD6126, but the response of the 4T1 tumors was unexpected at this tumor size. It has been reported that different tumors can have diverse responses to the same VDA treatment owing to inherent variations in their tumor microenvironments or vasculature. For example, upon administration of a single dose of CA4-P to breast adenocarcinoma CaNT tumors and the round cell sarcoma SaS, Parkins et al observed greater vascular damage in the tumors having higher nitric oxide synthase activity (33). While the functional vascular volume, assessed using a fluorescent carbocyanine dye, was significantly reduced at $18 \mathrm{~h}$ after CA4-P treatment in both tumor types, the degree of reduction was very different in the two tumors. Similarly, Skliarenko et al have reported that the KHT-C murine fibrosarcoma cell line and the CaSki human cervical carcinoma cell line responded differently to the same one time treatment with ZD6126 depending on their initial interstitial fluid pressures (IFP) (34). Notably, the two tumor types manifested IFP changes distinct from each other, and the tumors with higher IFPs had less tumor cell death. A single tumor can also have different responses to different VDAs. Dalal and Burchill treated TC-32 and A673 Ewing's sarcoma family of tumors with CA4-P, OXi4503, and OXi8007, and demonstrated that different VDAs can have varying efficacy and treatment results in a particular tumor type (35). In light of the previous results, it is not unreasonable that 4T1 and Caki-1 tumors could vary in their response to OXi4503, and that the differential response with tumor size reported with ZD6126 could be somewhat dissimilar with OXi4503. Further research is required to identify the conditions and mechanisms responsible for the differences in 4T1 and Caki-1 small tumor responses to OXi4503.

In summary, we used spectral imaging of tumor microvessel hemoglobin saturation with mouse window chamber tumors to measure the real-time response of 4T1 and Caki-1 tumors to repeat treatments with OXi4503. Some of the observations made with our imaging system were consistent with others in the literature obtained with different techniques, such as the timing of the onset of vascular changes induced after administration of the VDA. We also observed differences in the responses of the 4T1 and Caki-1 tumors. Specifically, the $4 \mathrm{~T} 1$ tumors were more responsive to treatment with OXi4503 than Caki-1 tumors in terms of permanent vascular damage and induction of tumor necrosis, a finding that contrasted with previous work using a different VDA. A better understanding of the mechanisms of action of VDAs and how they can vary across tumors or at different stages of tumor development may help optimize the application of these agents and improve their efficacy.

\section{Acknowledgements}

The authors acknowledge the assistance of Lori Rice, Ph.D., Chris Pampo and Sharon Lepler (Department of Radiation Oncology, University of Florida). Funding for this project was provided in part by US Public Health Service grant CA 084408 (DWS).

\section{References}

1. Denekamp J: Endothelial cell proliferation as a novel approach to targeting tumour therapy. Br J Cancer 45: 136-139, 1982.

2. Denekamp J, Hill SA and Hobson B: Vascular occlusion and tumour cell death. Eur J Cancer Clin On 19: 271-275, 1983.

3. Denekamp J and Hobson B: Endothelial cell proliferation in experimental tumours. Br J Cancer 46: 711-720, 1982.

4. Baguley BC: Antivascular therapy of cancer: DMXAA. Lancet Oncol 4: 141-148, 2003.

5. Chaplin DJ and Dougherty GJ: Tumor vasculature as a target for cancer therapy. Br J Cancer 80 (Suppl. 1): S57-S64, 1999.

6. Denekamp J: The tumor microcirculation as a target in cancer therapy: a clearer perspective. Eur J Clin Invest 29: 733-736, 1999.

7. Siemann DW: Vascular targeting agents. Horiz Cancer Ther 4: 4-14, 2002.

8. Siemann DW, Chaplin DJ and Horsman MR: Vascular-targeting therapies for treatment of malignant disease. Cancer 100: 2491-2499, 2004.

9. Thorpe PE: Vascular targeting agents as cancer therapeutics. Clin Cancer Res 10: 415-427, 2004.

10. Siemann DW, Bibby MC, Dark GG, Dicker AP, Eskens FALM, Horsman MR, Marme D and LoRusso PM: Differentiation and definition of vascular-targeted therapies. Clin Cancer Res 11: 416-420, 2005. 
11. Chan LS, Malcontenti-Wilson C, Muralidharan V and Christophi C: Alterations in vascular architecture and permeability following OXi4503 treatment. Anti-Cancer Drug 19: $17-22,2008$

12. Salmon HW and Siemann DW: Effect of the second-generation vascular disrupting agent OXi4503 on tumor vascularity. Clin Cancer Res 12: 4090-4094, 2006.

13. Sheng Y, Hua J, Pinney KG, Garner CM, Kane RR, Prezioso JA, Chaplin DJ and Edvardsen K: Combretastatin family member OXi4503 induces tumor vascular collapse through the induction of endothelial apoptosis. Int J Cancer 111: 604-610, 2004.

14. Siemann DW and Horsman MR: Vascular targeted therapies in oncology. Cell Tissue Res 335: 241-248, 2009.

15. Siemann DW, Chaplin DJ and Walicke PA: A review and update of the current status of the vasculature disabling agent combretastatin-A4 phosphate (CA4P). Expert Opin Inv Drug 18: 189-197, 2009.

16. El-Emir E, Geoffrey M, Boxer GM, Petrie IA, Boden RW, Dearling JL, Begent RH and Pedley RB: Tumour parameters affected by combretastatin A-4 phosphate therapy in a human colorectal xenograft model in nude mice. Eur J Cancer 41: 799-806, 2005

17. Galbraith SM, Maxwell RJ, Lodge MA, Tozer GM, Wilson J, Taylor NJ, Stirling JJ, Sena L, Padhani AR and Rustin GJS Combretastatin A4 phosphate has tumor antivascular activity in rat and man as demonstrated by dynamic magnetic resonance imaging. J Clin Oncol 21: 2831-2842, 2003.

18. Salmon HW, Mladinich C and Siemann DW: Evaluations of vascular disrupting agents $\mathrm{CA} 4 \mathrm{P}$ and OXi4503 in renal cell carcinoma (Caki-1) using a silicon-based microvascular casting technique. Eur J Cancer 42: 3073-3078, 2006.

19. Seshadri M, Spernyak JA, Maiery PG, Richard T. Cheneyz, Mazurchuk R and Bellnier DA: Visualizing the acute effects of vascular-targeted therapy in vivo using intravital microscopy and magnetic resonance imaging: correlation with endothelia apoptosis, cytokine induction, and treatment outcome. Neoplasia 9: 128-135, 2007.

20. Kotz B, West C, Saleem A, Jones T and Price P: Blood flow and $\mathrm{Vd}$ (water): both biomarkers required for interpreting the effects of vascular targeting agents on tumor and normal tissue. Mol Cancer Ther 8: 303-309, 2009.

21. Tozer GM, Akerman S, Cross NA, Barber PR, Björndahl M, Greco O, Harris S, Hill SA, Honess DJ, Ireson CR, Pettyjohn KL, Prise VE, Reyes-Aldasoro CC, Ruhrberg C, Shima DT and Kanthou C: Blood vessel maturation and response to vasculardisrupting therapy in single vascular endothelial growth factor-A isoform-producing tumors. Cancer Res 68: 2301-2311, 2008

22. DeDeugd C, Wankhede M and Sorg BS: Multimodal optical imaging of microvessel network convective oxygen transport dynamics. Appl Optics 48: D187-D197, 2009.
23. Sorg BS, Hardee ME, Agarwal N, Moeller BJ and Dewhirst MW: Spectral imaging facilitates visualization and measurements of unstable and abnormal microvascular oxygen transport in tumors. J Biomed Opt 13: article 014026, 2008.

24. Sorg BS, Moeller BJ, Donovan O, Cao Y and Dewhirst MW: Hyperspectral imaging of hemoglobin saturation in tumor microvasculature and tumor hypoxia development. J Biomed Opt 10: article 044004, 2005.

25. Kimura H, Braun RD, Ong ET, Hsu R, Secomb TW Papahadjopoulos D, Hong K and Dewhirst MW: Fluctuations in red cell flux in tumor microvessels can lead to transient hypoxia and reoxygenation in tumor parenchyma. Cancer Res 56: 5522-5528, 1996.

26. Shonat RD, Wachman ES, Niu W-H, Koretsky AP and Farkas DL: Near-simultaneous hemoglobin saturation and oxygen tension maps in mouse brain using an AOTF microscope. Biophys J 73: 1223-1231, 1997.

27. Jordan A, Hadfield JA, Lawrence NJ and McGown AT: Tubulin as a target for anticancer drugs: agents which interact with the mitotic spindle. Med Res Rev 18: 259-296, 1998.

28. Lin CM, Singh SB, Chu PS, Dempcy RO, Schmidt JM, Pettit GR and Hamel E: Interactions of tubulin with potent natural and synthetic analogs of the antimitotic agent combretastatin: a structure-activity study. Mol Pharmacol 34: 200-208, 1988.

29. Folkes LK, Christlieb M, Madej E, Stratford MRL and Wardman P: Oxidative metabolism of combretastatin A-1 produces quinone intermediates with the potential to bind to nucleophiles and to enhance oxidative stress via free radicals. Chem Res Toxicol 20: 1885-1894, 2007.

30. Salmon BA and Siemann DW: Characterizing the tumor response to treatment with combretastatin A4 phosphate. Int J Radiat Oncol Biol Phys 68: 211-217, 2007

31. Tozer GM, Kanthou C and Bagule BC: Disrupting tumour blood vessels. Nat Rev Cancer 5: 423-435, 2005.

32. Siemann DW and Rojiani AM: The vascular disrupting agent ZD6126 shows increased antitumor efficacy and enhanced radiation response in large, advanced tumors. Int J Radiat Oncol Biol Phys 62: 846-853, 2005.

33. Parkins CS, Holder AL, Hill SA, Chaplin DJ and Tozer GM: Determinants of anti-vascular action by combretastatin A-4 phosphate: role of nitric oxide. Br J Cancer 83: 811-816, 2000.

34. Skliarenko JV, Lunt SJ, Gordon ML, Vitkin A, Milosevic M and Hill RP: Effects of the vascular disrupting agent ZD6126 on interstitial fluid pressure and cell survival in tumors. Cancer Res 66: 2074-2080, 2006.

35. Dalal S and Burchill SA: Preclinical evaluation of vasculardisrupting agents in Ewing's sarcoma family of tumours. Eur J Cancer 45: 713-722, 2009. 